Informes de la Construcción Vol. 67, 539, erroo1

julio-septiembre 2015

ISSN-L: 0020-0883

\title{
FE DE ERRORES
}

Los autores del artículo:

Souto-Vidal, M., Ortiz-Sanz, J., Gil-Docampo, M. (2015). Implementación del levantamiento eficiente de fachadas mediante fotogrametría digital automatizada y el uso de software gratuito. Informes de la Construcción, 67(539): e107, doi: http://dx.doi.org/10.3989/ic.14.098.

pertenecen al Departamento de Ingeniería Agroforestal de la Escuela Politécnica Superior - Universidad de Santiago de Compostela, Lugo (España). 


\title{
Implementación del levantamiento eficiente de fachadas mediante fotogrametría digital automatizada y el uso de software gratuito
}

\section{Implementation of efficient facades survey by structure from motion photogrammetry and use of free software}

\author{
M. Souto-Vidal ${ }^{(*)}$, J. Ortiz-Sanz ${ }^{(*)}$, M. Gil-Docampo ${ }^{(*)}$
}

\section{RESUMEN}

El levantamiento de fachadas es un proceso demandado en multitud de ámbitos como la planificación arquitectónica y el planeamiento. La fotogrametría ofrece gran precisión geométrica y nos permite visualizar la textura del objeto en cuestión. Con la aparición de software gratuito en este ámbito, ésta se convierte en una de las técnicas más asequibles. Para demostrar las posibilidades de esta metodología, se presentan aquí tres ejemplos de levantamientos de fachadas de distinta tipología, realizados con fotogrametría y mediante la utilización del software gratuito, utilizando para ello una cámara convencional sin calibrar.

Además se incluye un estudio en el que se muestra un análisis de la precisión alcanzable con este proceso, y una comparación directa con dos instrumentos topográficos convencionales. El trabajo realizado muestra, por tanto, como la fotogrametría de objeto cercano posibilita el levantamiento de fachadas a un bajo coste y de una forma ágil, precisa y eficiente.

Palabras clave: Fotogrametría de objeto cercano; software gratuito; fachada; modelo tridimensional; ortofotografía.

\section{ABSTRACT}

The survey of facades is a defendant process in several areas like architectural design and planning. The photogrammetry provides high geometric accuracy and but allows us to see the texture of the object. With the emergence of free software in this area, it turns into one of the most accessible techniques. To demonstrate the potential of this technique, we present here three examples of facade surveys of different types, made with this technique and using free software, using for that an uncalibrated conventional camera.

It also includes a study that shows an analysis of the accuracy achievable with this process, and a direct comparison with two conventional surveying instruments. The work shows, therefore, as close-range photogrammetry allows a cheaper and quick, accurate and efficient survey of facades.

Keywords: Close range photogrammetry; free software; façade; three-dimensional model; orthophotography.

(*) Escuela Politécnica Superior - Universidad de Santiago de Compostela, Lugo (España).

Persona de contacto/Corresponding author: martinsoutovidal@gmail.com (M. Souto-Vidal).

Cómo citar este artículo/Citation: Souto-Vidal, M., Ortiz-Sanz, J., Gil-Docampo, M. (2015). Implementación del levantamiento eficiente de fachadas mediante fotogrametría digital automatizada y el uso de software gratuito. Informes de la Construcción, 67(539): e107, doi: http://dx.doi.org/10.3989/ic.14.098.

Licencia / License: Salvo indicación contraria, todos los contenidos de la edición electrónica de Informes de la Construcción se distribuyen bajo una licencia de uso y distribución Creative Commons Reconocimiento no Comercial 3.o. España (cc-by-nc). 


\section{INTRODUCCIÓN}

La metodología en el registro de información espacial está cambiando en los últimos años. La documentación de elementos constructivos por métodos de medición tradicionales de forma directa está siendo sustituida por métodos indirectos como la fotogrametría o la tecnología láser. La fotogrametría digital automatizada nos ofrece grandes posibilidades en este aspecto ya que nos proporciona gran calidad métrica y visual a bajo coste. La aparición de software gratuito o software de bajo coste disponible para hardware convencional ha hecho que la fotogrametría deje de estar al alcance de unos pocos (1) y haya aumentado su competitividad frente a las técnicas convencionales o las nuevas técnicas láser.

La técnica de la fotogrametría permite obtener una réplica digital a partir de la toma de una serie de fotografías tomadas desde diversos puntos de vista con un recubrimiento común. Las nuevas herramientas fotogramétricas identifican de forma automática puntos homólogos en distintas imágenes lo cual posibilita la orientación relativa de las mismas, es decir, permite determinar su posición espacial en el momento de la toma fotográfica. A partir de la nube de puntos generada a partir de estas fotos orientadas, se crea una superficie mediante triangulación, que posteriormente se texturiza. Esta automatización en el proceso la convierte en una técnica atractiva en diversos campos de aplicación, como arqueología (2) (3), patrimonio (4), arquitectura (5), planificación urbana (6), el estudio o simulación de desastres naturales (7) o morfogénesis (8). Además, una vez obtenido el modelo tridimensional, éste se puede ortoproyectar sobre un plano, lo cual permite obtener una ortoimagen sobre la cual se pueden digitalizar aquellos elementos de interés mediante cualquier herramienta de CAD. La necesidad de dotar a esta técnica de mayor competitividad en el mercado actual hace necesaria una revisión de las herramientas de software de bajo coste a nuestro alcance. Algunos autores han realizado modelos 3D mediante software gratuito obteniendo buenos resultados (9) (10) (11) (12). Actualmente existen varias herramientas que permiten obtener, de forma gratuita, una nube de puntos con coordenadas $\mathrm{x}, \mathrm{y}, \mathrm{z}$, a partir de la toma de fotografías en dos dimensiones. Entre éstas podemos citar fundamentalmente $\mathrm{ARC} 3 \mathrm{D}$ (2005) (13), Hypr3D (2010) (14), My3DScanner (2010) (15), Photosynth (2008) (16). A menudo estas herramientas están enfocadas hacia la réplica digital de pequeños objetos con la finalidad de su impresión en $3 \mathrm{D}$ o de su inclusión en visores web, por lo que pese a ser visualmente atractivos, su precisión geométrica merma a gran escala.

A menudo, el acceso a determinadas fachadas entraña cierta dificultad para el operario, ya sea por riesgo de derrumbe o por estar en un lugar inaccesible. Algunos autores han publicado artículos (17) (18), en los que se desarrolla un método para evitar estas dificultades. Así, mediante un medidor láser, pueden conocer la distancia al objeto sin necesidad de estar en contacto con él, permitiendo el escalado del modelo. Este proceso no sólo ahorra costes, sino que supone un procedimiento más seguro para el operario y elimina la posibilidad de cometer errores groseros. Sin embargo, este sistema no es óptimo a distancias grandes, ya que la visibilidad del láser puede inducir a error.

La utilización del croquis o la fotografía ayudados de medidas directas tomadas con flexómetro, distanciómetro laser o estación total sigue siendo útil hoy en día, sin embargo la posibilidad de obtener un modelo tridimensional de una forma rápida y de buena calidad métrica puede resultar muy útil en numerosos casos, pues el modelo tridimensional conserva tanto la geometría original como la textura. Ambos aspectos facilitan la determinación de deformaciones y agrietamientos en el inmueble, la aparición de humedades, etc. Factores necesarios en un proceso de restauración y rehabilitación. La fotogrametría de objeto cercano ya ha sido ampliamente utilizada y contrastada con software propietario para el levantamiento de fachadas, como por ejemplo en (19) (20) (21), en este trabajo se evaluarán las posibilidades de utilización de software fotogramétrico gratuito para dicho fin. Para ello se realizarán una serie de levantamientos de diversa dificultad y tipología, con una cámara convencional y utilizando dicho software. A partir de estos levantamientos se determinarán los siguientes aspectos: calidad de medida, versatilidad de la técnica, simplicidad (equipo necesario, coste del mismo, tiempo y tipo de supervisión necesaria del operador, tiempo de aprendizaje, dificultades en la toma de fotos, tiempo de procesado, necesidades de memoria), y la utilidad del producto obtenido, (posibilidades de obtener el modelo 3D y calidad visual del mismo, posibilidades de visualización, de exportación a otros formatos, de obtención de la ortofoto y de obtención el plano restituido de la fachada). Los resultados alcanzados proporcionarán información que facilitará la toma de decisiones para optar entre software propietario o software gratuito en función de las precisiones requeridas y el presupuesto disponible.

\section{METODOLOGÍA}

Para poder evaluar todos los aspectos previstos, se llevaron a cabo tres levantamientos de campo. El primero de ellos se orientó a la evaluación de la calidad de medida obtenida, mientras el segundo y tercero se plantearon para complementar la evaluación del resto de objetivos. Para ello se seleccionaron tres fachadas diferentes, cada una de ellas con unas características y una geometría que permitan evaluar cada uno de los objetivos propuestos.

El equipo básico utilizado en esta investigación se compone de una cámara digital réflex, modelo Canon EOS 500D, la cual dispone de una resolución máxima de 15,1 megapíxeles ( $4.752 \times 3.168$ píxeles) y una focal de $20 \mathrm{~mm}$; y un jalón topográfico con subdivisiones cada $10 \mathrm{~cm}$.

El software fotogramétrico básico utilizado en este estudio es el 123D Catch, este programa, en su versión original llamado Proyecto Photofly, se presentó en 2011 (22), es totalmente gratuito y su funcionamiento es muy sencillo. Este software está basado en SfM (Structure from Motion), el cual determina de forma automática, los parámetros de orientación interna de la cámara, lo que hace innecesaria una metodología expresa de calibración. Esta orientación se realiza en la nube, es decir, en el servidor propio del programa por lo que el tiempo de obtención del modelo tridimensional está íntimamente relacionado con el volumen de datos y la velocidad de la conexión a internet, y no tanto con la complejidad del objeto de estudio. Posteriormente se utiliza el software Blender (23), un software libre de edición 3D que permite obtener ortofotos a partir del modelo, y el software DraftSight (24), un software gratuito de CAD con el que se puede vectorizar la ortofoto para obtener los planos. 
Debido a que el procesado de las fotografías se realiza de forma automática, el usuario sólo puede condicionar la calidad del trabajo en la toma de las imágenes, por lo que este proceso es de vital importancia. La utilización en fotogrametría de cámaras no profesionales requiere un gran conocimiento de las posibilidades de uso de las mismas. En este aspecto, y pese al tiempo transcurrido desde su publicación, se deben tener en cuenta las Reglas 3x3 para la documentación patrimonial del CIPA (Comité Internacional de Fotogrametría Arquitectónica) (25).

$\mathrm{Al}$ igual que en cualquier otro trabajo de campo, la planificación previa es muy importante. En este caso se optó por realizar las fotografías en días nublados y a primera horas de la mañana. Esto se realizó para evitar sombras en las fotografías, no sólo porque degradan la calidad visual del modelo, sino porque su movimiento puede introducir errores en la orientación. El movimiento del sol puede provocar sombras en posiciones ligeramente distintas en las fotos de un mismo proyecto, lo cual puede dificultar la orientación de las imágenes y mermar la precisión geométrica.

En un principio el software de fotogrametría de objeto cercano trabajaba con fotografías convergentes (se buscaba la convergencia ideal de $90^{\circ}$ entre haces para determinar la posición con mucha precisión) y requería una geometría muy estricta en la toma de fotos para que funcionara el programa. El programa 123 Dcatch también trabaja a partir de haces convergentes, pero la mejora de los algoritmos de reconocimiento de la escena del software de fotogrametría digital automatizada permite que se utilicen más fotografías pero de forma que la geometría de las mismas sea más libre. 123Dcatch ajusta la posición de cada punto en todas las fotografías en las que sea visible y reajusta todo ese conjunto continuamente (y con el resto de fotos) hasta que consigue una solución de error mínimo. Además, para definir las nubes de puntos de la superficie del elemento, compara múltiples fotos con estereoscopía (fotografías que recogen una misma parte del elemento, por pequeña que sea). Así, para objetos de pequeñas dimensiones, la captura de fotos deberá hacerse apuntando al centro del objeto y rodeándolo si es posible. En el presente trabajo, debido al tamaño del objeto y a las limitaciones en cuanto a separación para el fotografiado por situarse las fachadas en calles, la toma fotográfica se realizó con el eje de la cámara perpendicular a la fachada. Posteriormente se realizaron barridos a mayor altura en aquellos casos en los que no se disponía de suficiente distancia de fotografiado.

Para la toma de fotografías se seleccionaron los siguientes parámetros:

- Se realizaron las fotografías en formato JPEG y con la mayor resolución de imagen disponible, pese a que el software fotogramétrico redimensiona en postproceso las imágenes a 3,6 megapíxeles para su orientación.

- Una apertura de diafragma pequeña, en este caso F8-F11, lo que hace los rayos incidentes sean más rectos y directos aumentando la profundidad de campo, es decir, la nitidez de la imagen. Este valor no se aumenta más para evitar posibles problemas ocasionados por la difracción.

- La sensibilidad del sensor o valor ISO se bajó al máximo, en este caso ISO10o, ya que esta opción permite la mayor resolución posible. Esta opción requiere una buena iluminación, lo que obliga a emplear el modo de prioridad de apertura Av (Apertura Value) que determinará automáticamente la velocidad de obturación.
- Se ajustó la cámara para que las fotografías estuvieran ligeramente sobreexpuestas, utilizando el histograma para determinar en cada foto el grado de corrección. Esto aumenta el contraste de nuestras imágenes y facilita la creación de una nube más densa. Para evitar trepidaciones, se utilizó trípode con el autodisparador de la cámara.

\subsection{Levantamiento de fachada moderna: determinación de la calidad de medida}

Como primer objeto de estudio se seleccionó una fachada que corresponde a una vivienda residencial situada en la Rúa de Viena, en Santiago de Compostela (UTM 538176, 4747894), cuyas dimensiones son 20,7 metros de ancho por 12 metros de alto. La elección de la fachada se debe a que posee elementos industrializados, regularmente distribuidos por la fachada. Lo que garantiza unas dimensiones muy uniformes en todos los elementos con variaciones dimensionales entre ellos muy por debajo de la resolución a la que se puede llegar con el equipo que se pretende contrastar. Por lo que se pueden tomar como medidas de referencia para el contraste de la calidad de medida del equipo fotogramétrico.

La primera parte del trabajo consistió en la medición de dos ventanas, utilizando un flexómetro de clase II de una longitud de $3 \mathrm{~m}$ cuyas desviaciones por temperatura fueron consideradas, y se determinaron las longitudes de los lados y las diagonales, dimensiones que se consideraron como referencia. Hecho esto se realizó un levantamiento fotogramétrico completo de la fachada descrita a una distancia de 5,10 y 20 metros, lo que supone una relación altura de la fachada-distancia de fotografiado de 1/2.4, 1/1.2 y 1/o.6 respectivamente. Estas distancias se seleccionaron para simular la situación de muchas calles estrechas con edificios altos y mostrar cómo condiciona la distancia de fotografiado en la precisión geométrica. Sobre estos levantamientos se determinaron las dimensiones de las ventanas a fin de realizar la comparación con las obtenidas con el flexómetro.

En la segunda parte del experimento, se determinaron una serie de medidas de distinta longitud distribuidas arbitrariamente por la fachada, como muestra la Figura 1b, aprovechando vértices y particularidades propias de la fachada que permitieran identificar de forma inequívoca los puntos a analizar sin necesidad de colocación de marcas o dianas externas, a fin de obtener una comparación directa con dos instrumentos topográficos contrastados, y así evaluar la calidad de la medida. El empleo de dianas artificiales no redundaría en una mayor precisión en el análisis de la calidad métrica para las fotografías de las fachadas objeto del estudio, dadas las herramientas del software de fotogrametría para la identificación de los puntos (Figura 4a) y la resolución de las fotos que éste emplea.

El primer instrumento utilizado fue una estación total modelo Topcon 3007 cts con una precisión en la medición sin prisma de $10 \mathrm{~mm}$ en distancias comprendidas entre 1,5 y $25 \mathrm{~m}$ y el segundo, un distanciómetro láser modelo Leica Distimax 100.

Una vez realizadas las fotografías se introdujeron en el 123D Catch, el cual calcula de forma automática la posición relativa de las cámaras en el momento de la toma mediante la identificación de píxeles homólogos en imágenes diferentes. Con lo que, a diferencia de otras herramientas similares, el proceso de calibración y cálculo de la focal se automatiza por completo. De esta forma se realizó el levantamiento fotogramétrico, siguiendo las pautas de fotografiado descritas anteriormente e 


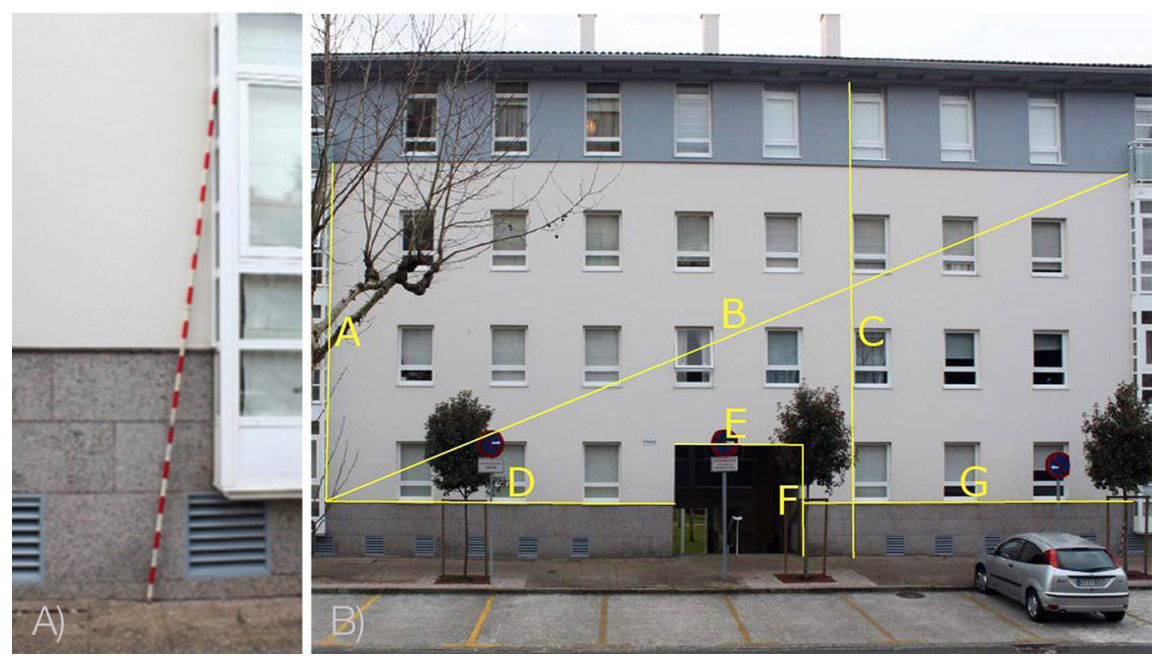

Figura 1. a) Jalón topográfico utilizado para escalar los modelos y

b) Muestra de las mediciones seleccionadas para la comparación entre los instrumentos topográficos y la fotogrametría.

introduciendo en la escena un jalón topográfico (Figura 1a), el cual nos permite tener una distancia de referencia conocida en el modelo tridimensional y así disponer de un modelo métrico.

La obtención de las diagonales de las ventanas que conforman la fachada se realiza de forma manual, para ello se restituyen los puntos a través de su determinación en un mínimo de tres imágenes. Todas estas diagonales se compararon con la medición directa realizada con un flexómetro, y se determinan una serie de cálculos estadísticos: error en la medida, media del error, desviación típica, media del error en valor absoluto, desviación típica del error en valor absoluto y el error medio cuadrático.

Para validar en qué medida condiciona el proceso de escalado del modelo en la precisión final, se restituyeron sobre el modelo los puntos que forman las subdivisiones del jalón previamente introducido en la escena (cuyas medidas reales son conocidas) y mediante el cual le asignamos la longitud real con el software 123D Catch, que nos permite tener un modelo 3D métrico. De igual forma, se restituyeron sobre el mismo modelo utilizado en el análisis de la precisión local, los puntos seleccionados en la segunda parte del experimento, y se compararon con las medidas obtenidas tanto por la estación total como por el distanciómetro láser. Para la medición con estación total se estacionó a una distancia aproximada de $25 \mathrm{~m}$, en un punto arbitrario, desde el cual se veía la totalidad de la fachada y se podían visar todos los puntos con comodidad. Desde ese punto se realizó una radiación utilizando el láser del aparato. Las mediciones por tanto, se obtuvieron por diferencia de coordenadas. Con el distanciómetro láser se realizaron las mismas medidas que con la estación total, sin embargo debido a la posición de éstas, no siempre se pudo utilizar el instrumento apoyado en un trípode. Por lo que se optó por realizar cada medición tres veces y luego hacer el promedio. Con estas mediciones se realizó la comparación entre los datos obtenidos por fotogrametría y los datos obtenidos por la estación total y el distanciómetro. Repitiendo los cálculos estadísticos realizados en la primera parte.

\subsection{Levantamiento de fachada en un casco histórico}

En el segundo caso se realizó el levantamiento fotogramétrico de una fachada de una vivienda residencial en el casco viejo de Santiago de Compostela (Rúa Nova) (UTM: 537280, 4747466), con el objetivo de obtener un plano y una ortofoto de la misma.

La fachada tiene 9 metros de alto por 7 de ancho (Figura 2.a). La particularidad de este caso radica en su geometría; cuenta con un soportal en la planta baja, como es común en la tipología de la zona, en la primera planta posee una pequeña terraza con vegetación y en la segunda planta un escudo heráldico, de un posible interés patrimonial. Estos detalles pueden dificultar la ortorrectificación de la proyección cónica e imposibilitar obtener una ortofoto verdadera.

El fotografiado se realizó siguiendo las pautas ya descritas e introduciendo un jalón en la escena, bastando un total de 22 fotografías para cubrir el total de la fachada. Una vez orientadas estas imágenes en el 123D Catch se obtiene un modelo tridimensional, formado por una malla de triángulos que puede ser visualizada en tres grados de densidad (Figura 3) a esta malla de triángulos se le asigna la textura obteniendo el modelo tridimensional (Figura 2.b). Este se escaló mediante la asignación del valor real de la longitud del jalón sobre el modelo. Además se definió el eje de coordenadas $x, y, z$, aprovechando la geometría propia del edificio.

A partir de aquí se procede a la restitución manual de los puntos y líneas que permitan definir la geometría de la fachada, mediante la identificación de puntos homólogos (Figura 4a) en un mínimo de tres imágenes. Sin embargo, como en este caso la fachada disponía de multitud de detalles, sólo se determinaron una serie de puntos en lugares fácilmente identificables.

A partir de este momento, se utilizan dos herramientas más de software gratuito. Por una parte se exporta el modelo al software Blender en formato Wavefront (.obj); esta elección se debe a que este formato conserva la geometría y la textura original del objeto. La necesidad de utilización de este software radica en que éste dispone de visor ortográfico como se muestra en la Figura 4b, herramienta del cual carece el 123D Catch, y que nos permite obtener una ortofotografía de la fachada. Por otra parte se exportan los puntos y líneas al software de diseño DraftSight, en formato .dwg. 

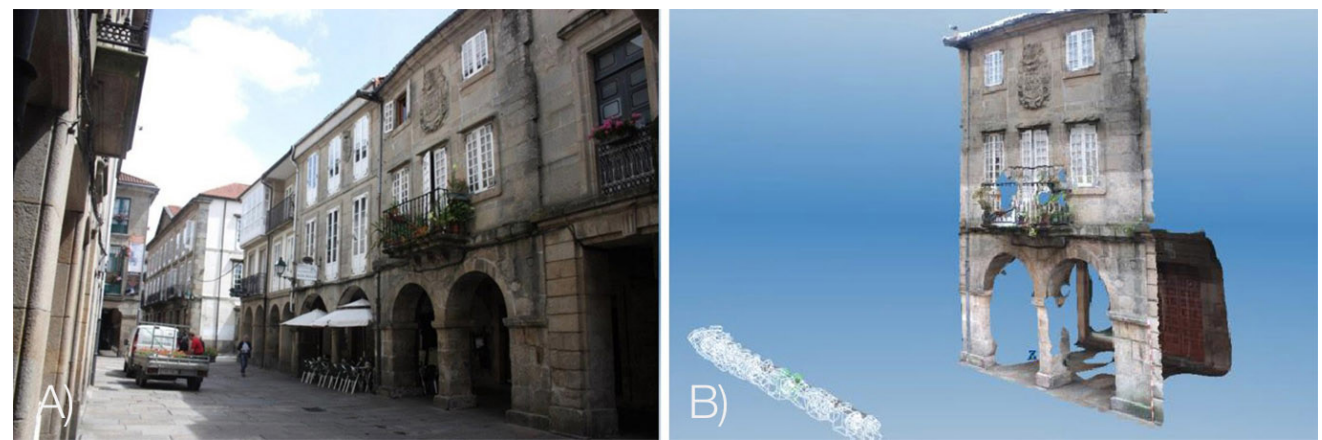

Figura 2.a) Fachada situada en Rúa Nova (Santiago de Compostela) y b) modelo 3D obtenida con 123D Catch.
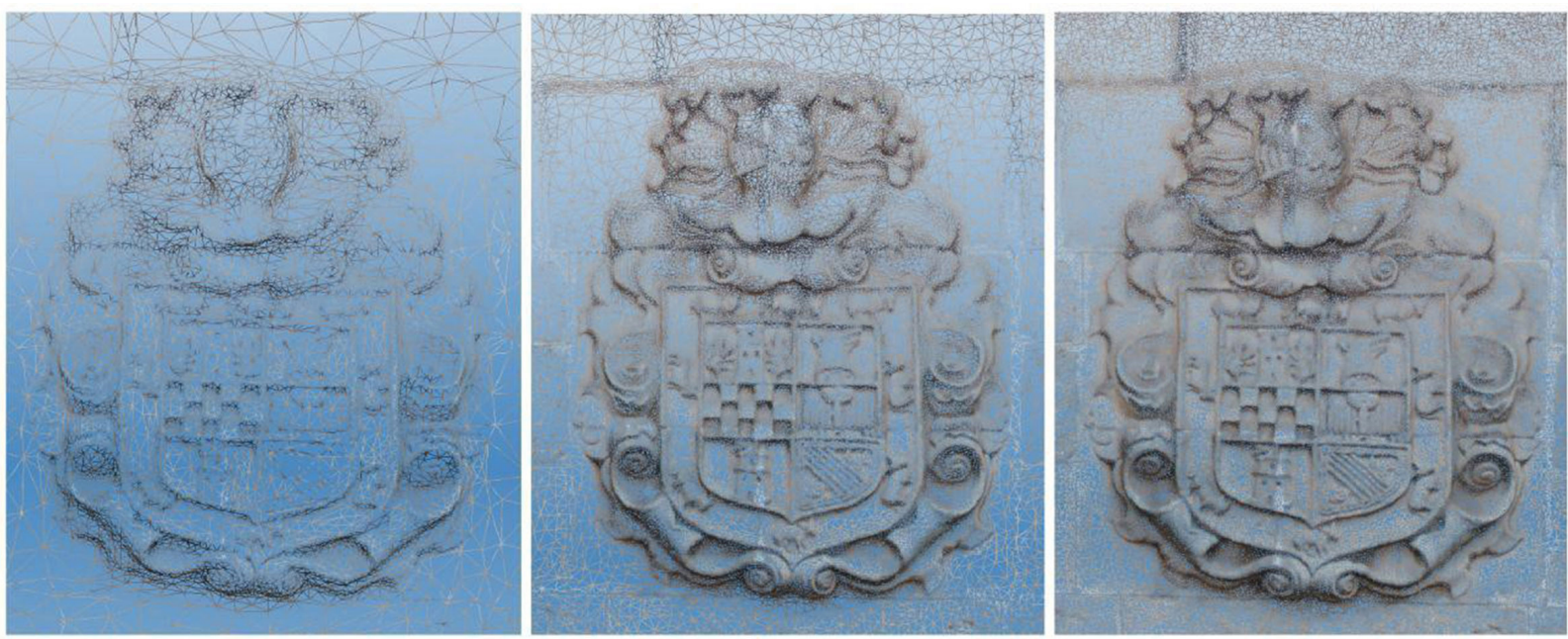

Figura 3. Muestra de los tres grados de densidad que nos permite el software 123D Catch.
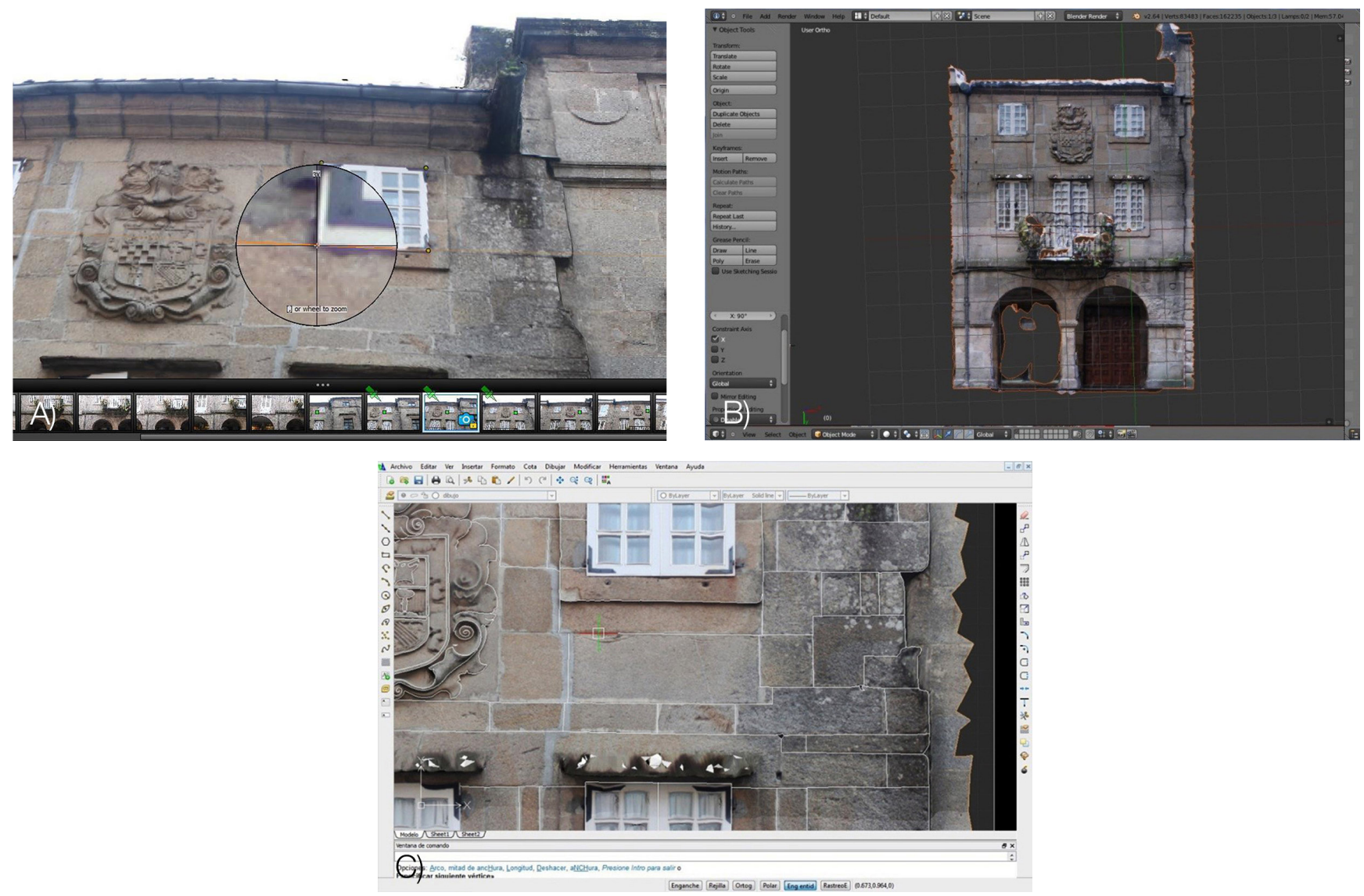

Figura 4. a) Restitución manual de elementos con 123D Catch, b) Visor ortográfico en Blender y c) Proceso de vectorización en DraftSight. 
Esta ortofotografía se inserta en el DraftSight y se escala y orienta mediante los puntos restituidos manualmente en el 123D Catch. De esta forma se puede hacer una comprobación de la captura de la ortofoto, pues la posición de todos los puntos de control debe ser exacta sobre la imagen. En este software se puede obtener un plano rápidamente digitalizando los elementos destacables sobre la ortoimagen como se muestra en la Figura 4c, o presentar directamente ésta, en función de las necesidades del momento.

\subsection{Levantamiento de fachada exenta}

Y el tercer experimento en campo consistió en el levantamiento fotogramétrico de la fachada principal de la Iglesia de San Miguel en la parroquia de Orbazai, situada en Lugo (UTM: 614397, 4762555).

La particularidad de este caso radica en la poca distancia disponible para fotografiar la fachada, la cual imposibilita captar todos los detalles (Figura 5a). Este problema es común en el levantamiento de fachadas en entornos urbanos, sobre todo en cascos históricos. La relación altura-anchura de calle es 1/3.2 pudiéndose considerar un caso extremo.

El objetivo de este levantamiento es la obtención de la planimetría de la fachada. El proceso se subdividió en dos partes, se realizó una primera sesión en el que tomaron 43 fotografías con trípode, cubriendo el total de la fachada. Sin embargo, debido a la poca distancia cámara-objeto de la que se disponía, en la parte superior no se alcanzaba a ver todos los detalles del campanario de la iglesia. Por lo que se decidió hacer otra sesión de fotografías esta vez sin trípode, con el objetivo de tomar las imágenes lo más alto posible. Para evitar la trepidación se abrió más el diafragma dejando pasar más luz al sensor y realizando las fotografías más rápido. En este caso se realizaron 26 fotografías más de la parte superior de la fachada, que se añadieron al procesado en 123D Catch. El proceso posterior es análogo al descrito en el caso anterior, esto es: generación de la ortofoto con el software Blender y digitalización del plano con Draghtsight.

\section{RESULTADOS}

En este apartado se muestran los resultados obtenidos en los cálculos estadísticos realizados a partir de los datos del primer levantamiento fotogramétrico realizado, así como productos gráficos obtenidos en los otros casos.

El análisis de la calidad de medida realizado se divide en tres partes; una primera parte en la que se analiza la precisión local del modelo, una segunda parte en la que se observa cómo influye el proceso de escalado utilizado en la precisión y finalmente la tercera parte en la que se realiza una comparación directa con dos instrumentos comunes en topografía.

De esta forma, el primer levantamiento a una distancia de 5 metros no fue satisfactorio debido a que el software no consiguió realizar la orientación de las fotografías. Esto se puede deber principalmente a dos factores, por una parte al patrón geométrico repetitivo, y por otra parte el fotografiado de superficies especulares. En este caso, el levantamiento de una fachada con ventanas iguales confunde al programa a la hora de la orientación, así como los reflejos ocasionados por las mismas. Estas fotografías no serían desechables, pues el software permite la orientación manual de las fotografías, sin embargo este estudio se pretende determinar la precisión proporcionada por el software de forma automática. Los levantamientos a 10 y 20 metros sí fueron satisfactorios, mostrándose en las Tablas 1 y 2.
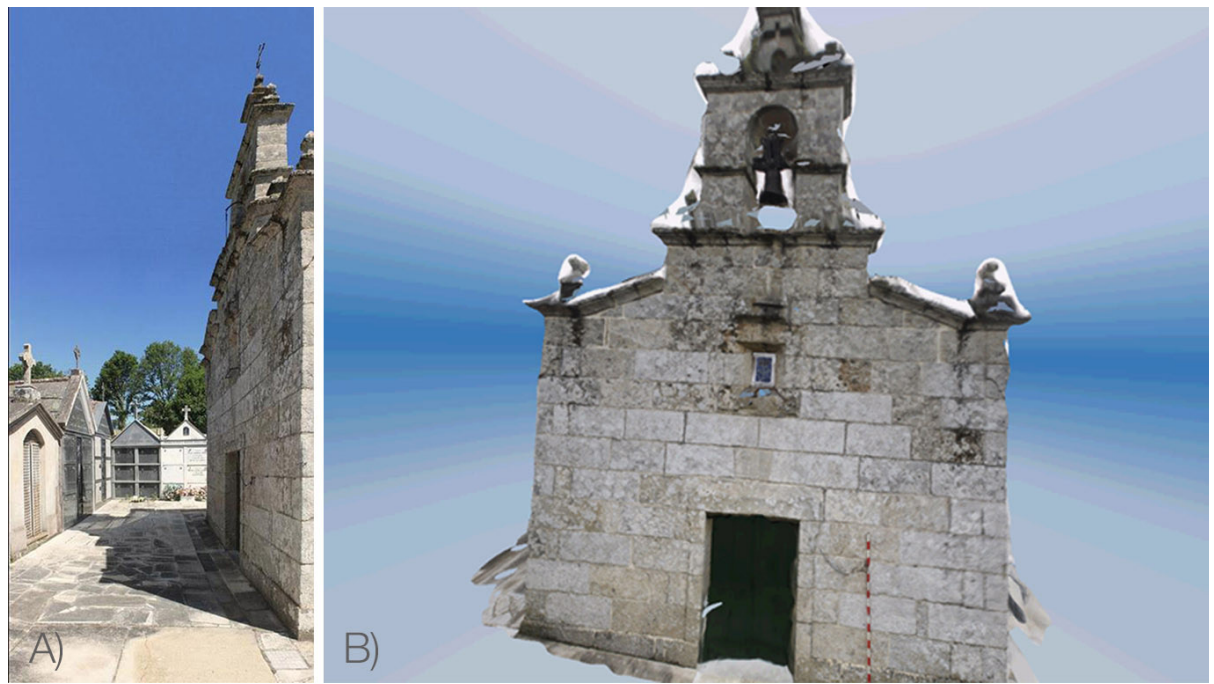

Figura 5.a) Fachada principal de la Iglesia de San Miguel en la parroquia de Orbazai y b) Modelo 3D de la misma

Tabla 1. Resultados obtenidos a partir del estudio de las diagonales obtenidas con fotogrametría a 10 y 20 metros.

\begin{tabular}{|c|c|c|c|c|c|c|c|}
\hline $\begin{array}{c}\text { Distancia de } \\
\text { fotografiado }\end{array}$ & $\begin{array}{c}\text { Error } \\
\text { máximo } \\
\mathbf{( m )}\end{array}$ & $\begin{array}{c}\text { Error } \\
\text { mínimo } \\
\mathbf{( m )}\end{array}$ & $\begin{array}{c}\text { Media } \\
\mathbf{e r r o r} \\
\mathbf{( m )}\end{array}$ & $\begin{array}{c}\text { Desv. típica } \\
\mathbf{e r r o r} \\
\mathbf{( m )}\end{array}$ & $\begin{array}{c}\text { Media error } \\
\text { v. absoluto } \\
\mathbf{( m )}\end{array}$ & $\begin{array}{c}\text { Desv. típica error } \\
\text { v. absoluto } \\
\mathbf{( m )}\end{array}$ & $\begin{array}{c}\text { RMSE } \\
\mathbf{( m )}\end{array}$ \\
\hline 10 & 0,034 & $-0,043$ & $-0,002$ & 0,017 & 0,013 & 0,01 & 0,017 \\
\hline 20 & 0,057 & $-0,042$ & 0,007 & 0,018 & 0,015 & 0,012 & 0,019 \\
\hline
\end{tabular}


Para observar en qué medida repercute en la precisión el escalar un modelo completo de una fachada a través de la asignación de una medida real a un objeto de pequeña dimensión introducido en la escena, se realizó un análisis estadístico en función de las subdivisiones de un jalón topográfico (Tabla 3).
Y la síntesis de la comparativa entre las mediciones determinadas por fotogrametría y topografía se recoge en la Tabla 4.

Por otra parte en la Figura 6 se muestran los documentos gráficos de las fachadas propuestas.

Tabla 2. Resultados obtenidos a partir del estudio de las diagonales obtenidas con fotogrametría a 10 y 20 metros en función de la diagonal de la escena.

\begin{tabular}{|c|c|c|c|c|c|c|c|}
\hline $\begin{array}{c}\text { Distancia de } \\
\text { fotografiado } \\
\mathbf{( m )}\end{array}$ & $\begin{array}{c}\text { Error } \\
\text { máximo } \\
\mathbf{( m )}\end{array}$ & $\begin{array}{c}\text { Error } \\
\text { mínimo } \\
\mathbf{( m )}\end{array}$ & $\begin{array}{c}\text { Media } \\
\text { error } \\
\mathbf{( m )}\end{array}$ & $\begin{array}{c}\text { Desv. típica } \\
\text { error } \\
\mathbf{( m )}\end{array}$ & $\begin{array}{c}\text { Media error } \\
\text { v. absoluto } \\
\mathbf{( m )}\end{array}$ & $\begin{array}{c}\text { Desv. típica error } \\
\text { v. absoluto } \\
\text { (m) }\end{array}$ & $\begin{array}{c}\text { RMSE } \\
\text { (m) }\end{array}$ \\
\hline 10 & $1 / 659$ & $1 / 521$ & $1 / 13892$ & $1 / 1356$ & $1 / 1742$ & $1 / 2135$ & $1 / 1318$ \\
\hline 20 & $1 / 393$ & $1 / 533$ & $1 / 3315$ & $1 / 1237$ & $1 / 1518$ & $1 / 1793$ & $1 / 1179$ \\
\hline
\end{tabular}

Tabla 3. Resultados obtenidos a partir del estudio de las subdivisiones del jalón topográfico a 10 metros y 20 metros.

\begin{tabular}{|c|c|c|c|c|c|c|c|}
\hline $\begin{array}{c}\text { Distancia de } \\
\text { fotografiado } \\
\text { (m) }\end{array}$ & $\begin{array}{c}\text { Error } \\
\text { máximo } \\
\mathbf{( m )}\end{array}$ & $\begin{array}{c}\text { Error } \\
\text { mínimo } \\
\mathbf{( m )}\end{array}$ & $\begin{array}{c}\text { Media } \\
\text { error } \\
\mathbf{( m )}\end{array}$ & $\begin{array}{c}\text { Desv. típica } \\
\text { error } \\
\mathbf{( m )}\end{array}$ & $\begin{array}{c}\text { Media error } \\
\text { v. absoluto } \\
\mathbf{( m )}\end{array}$ & $\begin{array}{c}\text { Desv. típica error } \\
\text { v. absoluto } \\
\text { (m) }\end{array}$ & $\begin{array}{c}\text { RMSE } \\
\text { (m) }\end{array}$ \\
\hline 10 & 0,005 & $-0,004$ & 0,001 & 0,002 & 0,002 & 0,001 & 0,002 \\
\hline 20 & 0,006 & $-0,004$ & 0,001 & 0,003 & 0,003 & 0,002 & 0,003 \\
\hline
\end{tabular}

Tabla 4. Resultados obtenidos en la comparativa con la estación total y el distanciómetro, con el levantamiento fotogramétrico a 10 metros.

\begin{tabular}{|c|c|c|c|c|c|c|c|}
\hline $\begin{array}{c}\text { Distancia de } \\
\text { fotografiado } \\
\mathbf{( m )}\end{array}$ & $\begin{array}{c}\text { Error } \\
\text { máximo } \\
\mathbf{( m )}\end{array}$ & $\begin{array}{c}\text { Error } \\
\text { mínimo } \\
\mathbf{( m )}\end{array}$ & $\begin{array}{c}\text { Media } \\
\mathbf{e r r o r} \\
\mathbf{( m )}\end{array}$ & $\begin{array}{c}\text { Desv. Típica } \\
\mathbf{e r r o r} \\
\mathbf{( m )}\end{array}$ & $\begin{array}{c}\text { Media error } \\
\text { v. absoluto } \\
\mathbf{( m )}\end{array}$ & $\begin{array}{c}\text { Desv. Típica error } \\
\text { v. absoluto } \\
\mathbf{( m )}\end{array}$ & $\begin{array}{c}\text { RMSE } \\
\mathbf{( m )}\end{array}$ \\
\hline Estación total & $-0,035$ & 0,009 & $-0,018$ & 0,017 & 0,022 & 0,012 & 0,025 \\
\hline Distanciómetro & $-0,047$ & $-0,005$ & $-0,015$ & 0,023 & 0,024 & 0,013 & 0,027 \\
\hline
\end{tabular}

A)
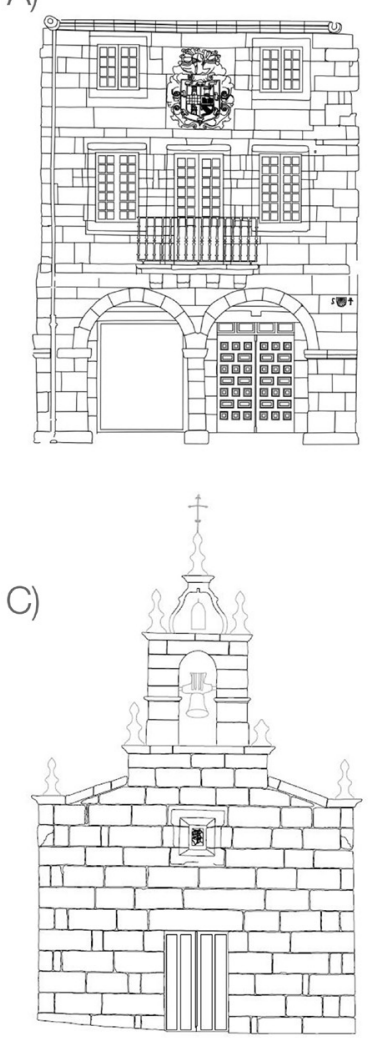

B)
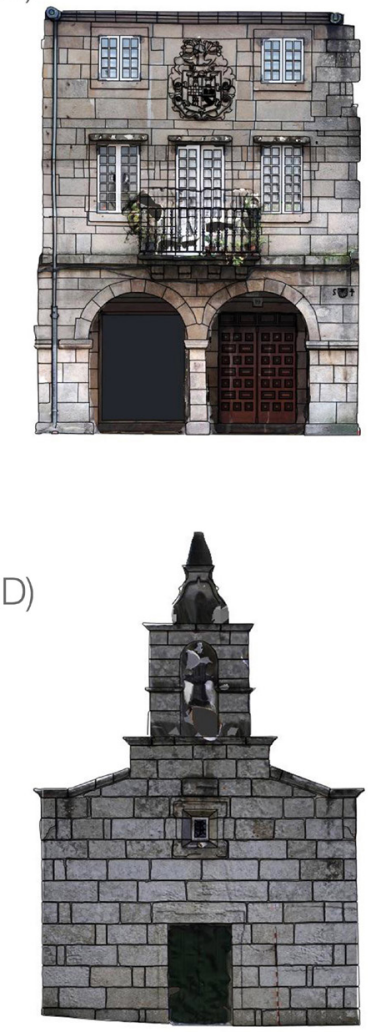

Figura 6. Planimetría y ortofoto de la fachada de un casco histórico (a y b) y de la iglesia de San Miguel (c y d). 


\section{DISCUSIÓN DE RESULTADOS}

La evaluación del presente trabajo se realizó en términos de 1) Calidad de medida, 2) Simplicidad/Coste y 3) Utilidad del producto obtenido en el estudio de las fachadas. A raíz de los resultados obtenidos se expone:

1) Calidad de medida: Los resultados del análisis de precisión a una distancia de 10 y 20 metros muestran una precisión aceptable, útil para multitud de trabajos pero inferior a la alcanzable con software comercial. Analizando las Tablas 1 y 2 observamos que el error medio es pequeño, sin embargo si se observa el error máximo y mínimo vemos que el rango de error es considerable en ambas distancias. Aunque los errores son mayores a 20 metros, si establecemos una relación entre el error medio cuadrático y la distancia de fotografiado se obtiene un error de $1 / 588$ a 10 metros y $1 / 1052$ a 20 metros.

Si comparamos los resultados obtenidos en las Tablas 1 y 3 en función del error medio cuadrático observamos que: a una distancia de $10 \mathrm{~m}$ se obtuvo un RMSE de $17 \mathrm{~mm}$ en la medición de las ventanas y un RMSE de $2 \mathrm{~mm}$ en la medición del jalón. Esta diferencia es lógica y muestra en que forma condiciona el método de escalado en el resultado final y cuestiona la metodología empleada. De igual forma que justifica las diferencias obtenidas con los instrumentos topográficos, mostrados en la Tabla 4. En la que el error medio está en torno a los $20 \mathrm{~mm}$, precisión que puede limitar la aplicabilidad de este software cuando se precisan escalas superiores a 1:100.

Para comparar los resultados obtenidos con otros autores debemos establecer un criterio común en la forma de presentar los datos. Para ello se establece una relación entre la diagonal del objeto de estudio y el valor de la desviación típica obtenido en la medida. De esta forma en (26) los autores obtienen, utilizando el mismo software, una precisión de 1/60o en la medición de una serie de puntos de control situados con estación total. Utilizando este criterio, se obtuvo un valor de $1 / 73$ en la medición de las diagonales de las ventanas y 1/602 en el jalón lo cual no hace sino corroborar lo dicho en el párrafo anterior.

Esta precisión también está condicionada por el tamaño reducido de las imágenes a procesar, (el software redimensiona imágenes superiores a 3,6 megapíxeles) lo cual dificulta la restitución de elementos a grandes distancias y constituye una de las principales limitaciones del software fotogramétrico empleado. Otros inconvenientes son la existencia de un límite en el volumen de datos a procesar, así como la restricción del formato de imagen de entrada (sólo permite imágenes en JPEG y TIFF). La utilización de las imágenes en un formato no comprimido mejoraría la calidad en todos los aspectos (27).

2) Simplicidad/Coste: La sencillez y versatilidad de este método es indiscutible. El manejo de la cámara fotográfica no requiere grandes conocimientos y los programas informáticos utilizados son muy intuitivos. Por lo que no se requiere una gran especialización por parte del usuario.

También debemos destacar la rapidez de esta técnica, se estima que el tiempo de fotografiado no supera los veinte minutos y su orientación con el software fotogramétrico no es superior a una hora. Aunque como ya se ha dicho, este tiempo variará en función de la complejidad del objeto de estudio y de la velocidad de la conexión a la red disponible.

El uso de una cámara convencional, no métrica y sin calibrar, y la utilización únicamente de software gratuito, hacen de esta técnica una de las más asequibles.

3) Utilidad del producto obtenido: este proceso nos proporciona tres productos diferentes.

En primer lugar el modelo (Figuras $2 b$ y 5 b) que es exportable a distintos formatos (.dwg, .fbx, .rzi, .obj, .ipm y .las), lo cual posibilita trabajar con la mayoría de los software de modelado tridimensional, tanto libre como propietario. Cabe destacar que el grado de detalle es inversamente proporcional a la manejabilidad del modelo y obliga a disponer de un mayor equipo informático.

En segundo lugar se obtienen las ortofotos (Figura 6), estas son de buena calidad, sin embargo, este producto está altamente condicionado con la distancia cámara-objeto, cuestión estudiada en el caso de la fachada de Orbazai, en el que no se pudo obtener la totalidad de los detalles en la parte superior de la misma. Este problema es independiente de la técnica planteada y la única solución pasa por tomar las fotografías a mayor altura, evitando así zonas ocultas en el modelo, cuestión que encarece el coste total de un proyecto. Esto perjudicó la calidad visual de ambos levantamientos, la presencia de salientes y marquesinas, imposibilitaron una restitución precisa de todos los elementos. De esta forma estos elementos se representaron de forma aproximada, especificándose con la correspondiente leyenda. Por otra parte, las fotografías se realizaron en condiciones de luz ideales, reduciendo considerablemente sombras en el producto final; por lo que no se evaluó esta técnica en condiciones de luz adversas (a contraluz, severos contrastes, etc.). La presencia de elementos ajenos a la fachada provoca ruido en el modelo y degrada la calidad visual de la ortofoto. Esto lo observamos en el caso del levantamiento de Santiago de Compostela, en el que la presencia de vegetación en la primera planta no sólo reduce la calidad de la ortofoto, sino que dificulta el proceso de obtención de la planimetría.

En tercer lugar se obtienen los planos, mediante esta técnica se pueden obtener grandes resultados como se muestra en la Figura 6. Con la restitución manual de elementos se puede conseguir gran precisión geométrica y restituir cualquier elemento captado en las imágenes permitiendo la obtención de un plano a medida de cada usuario, es decir, restituir sólo aquellos elementos que sean realmente de interés en función de la finalidad del proyecto. Además, existe la posibilidad de realizar el plano a partir de la digitalización de la ortofoto, de esta forma el tiempo de obtención del plano de fachada es considerablemente menor. Como se puede observar en el segundo caso realizado, se puede alcanzar gran detalle con esta técnica.

\section{CONCLUSIÓN}

Los levantamientos propuestos se realizaron satisfactoriamente mostrando una gran rapidez y versatilidad mediante el empleo de la fotogrametría. La facilidad y automatización del software fotogramétrico evaluado permite su utilización por usuarios sin alta especialización. Además se ha obteni- 
do documentación métrica con una textura fotorrealista de buena calidad, la cual es más fácilmente interpretable que los documentos cartográficos convencionales y pueden ser una potente herramienta en el ámbito arquitectónico y cultural.

El coste del equipo y la posibilidad de utilizar software gratuito aumenta el potencial de esta técnica. Sin embargo, estas herramientas muestran carencias en trabajos que requieran gran precisión, limitándolo a una serie de usos. Este trabajo muestra que el software fotogramétrico utilizado no está a la altura de las principales herramientas del mercado, aunque se considera totalmente válido para las aplicaciones propuestas.

No obstante, el análisis de los resultados en comparación con otros autores hace suponer que se podría alcanzar mayor precisión escalando el modelo a partir de la determinación de medidas sobre las fachadas de mayor longitud. Por otra parte, la continua aparición de nuevas herramientas de software así como el desarrollo de las mismas, hacen necesario un seguimiento en un futuro próximo.
De igual forma la obtención de modelos tridimensionales en formato digital, abre nuevas puertas a la forma en que consideramos el registro de la información geoespacial. Este formato es estable y preciso, de fácil distribución y visualización por gran número de dispositivos móviles. Además, no debemos olvidar que estas réplicas no solo registran la información espacial, sino que también registran un momento determinado del tiempo, convirtiéndolas en una herramienta ideal para cuantificar el deterioro y evolución de los inmuebles, ya sea debido a efectos naturales o antrópicos.

Por último, se debe destacar el potencial de este formato como soporte de nuevas técnicas basadas en visión computacional. El continuo avance de la informática hace que a día de hoy sea posible el desarrollo aplicaciones basadas en $V R$ y $A R$ (Virtual Reality y Augmented Reality), que optimizan los procesos de planificación y permite nuevas posibilidades en todo tipo de disciplinas, mediante la realización de hipótesis virtuales o simulaciones sobre los modelos digitales o sobre el propio medio físico.

\section{REFERENCIAS}

(1) Aguilar, M. A., Aguilar, F. J., Agüera, F. (2005). Mapping small areas using a low-cost close range photogrammetric software package with aerial photography. The Photogrammetric Record, 20(112): 335-350, doi: http://dx.doi.org/10.1111/ j.1477-9730.2005.00331.x.

(2) Ducke, B., Score, D., Reeves, J (2010). Multiview 3D reconstruction of the archaeological site at Weymouth from image series. Computers \& Graphics, 35(2): 375-382, doi: http://dx.doi.org/10.1016/j.cag.2011.01.006.

(3) De Reu, J., Plets, G., Verhoeven, G., De Smedt, P., Bats, M., Cherretté, B., De Maeyer, W., Deconynck, J., Herremans, D. Laloo, P., Van Meirvenne, M., De Clercq, W. (2012). Towards a three-dimensional cost-effective registration of the archaeological heritage. Journal of Archaeological Science, 40(2): 1108-1121, doi: http://dx.doi.org/10.1016/j. jas.2012.08.040.

(4) Portalés, C., Lerma, J. L., Navarro, S. (2010). Augmented reality and photogrammetry: A synergy to visualize physical and virtual city environments. ISPRS Journal of Photogrammetry and Remote Sensing, 65(1): 134-142, doi: http:// dx.doi.org/10.1016/j.isprsjprs.2009.10.001.

(5) Bellotti, F., Berta, R., Cardona, R., De Gloria, A. (2011). An architectural approach to efficient 3D urban modeling. Computers \& Graphics, 35(5): 1001-1012, doi: http://dx.doi.org/10.1016/j.cag.2011.07.004.

(6) Núñez, M.A., Buill, F. (2009). Evolution of the architectural and heritage representation. Landscape and Urban Planning, 91(2): 105-112, doi: http://dx.doi.org/10.1016/j.landurbplan.2008.12.006.

(7) Kanga, Z., Zhang, L., Zlatanova, S., Li, J. (2010). An automatic mosaicking method for building facade texture mapping using a monocular close-range image sequence. ISPRS Journal of Photogrammetry and Remote Sensing, 65(3): 282293, doi: http://dx.doi.org/10.1016/j.isprsjprs.2009.11.003.

(8) Stojakovic, V., Tepavcevic, B. (2011). Image-based modeling approach in creating 3D morphogenetic reconstruction of Liberty Square in Novi Sad. Journal of Cultural Heritage, 12(1): 105-110, doi: http://dx.doi.org/10.1016/j.culher.2010.06.001.

(9) Guarnieri, A., Pirotti, F. Vettore, A. (2010). Cultural heritage interactive 3D models on the web: An approach using open source and free software. Journal of Cultural Heritage, 11(3): 350-353, doi: http://dx.doi.org/10.1016/j.culher.2009.11.011.

(10) Koutsoudis, A., Arnaoutoglou, F., Chamzas, C. (2007). On 3D reconstruction of the old city of Xanthi. A minimum Budget approach to virtual touring based on photogrammetry. Journal of Cultural Heritage, 8(1): 26-31, doi: http://dx.doi. org/10.1016/j.culher.2006.08.003.

(11) Falkingham, P. L. (2012). Acquisition of high resolution three-dimensional models using free, open- source, photogrammetric software. Palaentologia Electronica, 15(1): 15. http://palaeo-electronica.org/content/issue1-2012technicalarticles/92-3d-photogrammetry.

(12) Bhatla, A., Choe, S. Y., Fierro, O., Leite, F. (2012). Evaluation of accuracy of as-built 3D modeling from photos taken by handheld digital cameras. Automation in Construction, 28: 116-127, doi: http://dx.doi.org/10.1016/j.autcon.2012.06.003.

(13) Automatic Reconstruction Cloud. http://www.arc3d.be/.

(14) Hypr 3D. http://www.hypr3d.com/

(15) My3DScanner. http://www.my3dscanner.com/.

(16) Microsoft Photosynth. http://photosynth.net/.

(17) Rodríguez, J., Martín, M. T., Arias, P., Ordóñez, C., Herráez, J. (2008). Flat elements on buildings using close-range photogrammetry and laser distance measurement. Optics and Lasers in Engineering, 46(7): 541-545, doi: http://dx.doi. org/10.1016/j.optlaseng.2008.02.007. 
(18) Ordóñez, C., Martínez, J., Arias, P., Armesto, J. (2010). Measuring building façades with a low-cost close-range photogrammetry system. Automation in Construction, 19(6) : 742-749, doi : http://dx.doi.org/10.1016/j.autcon.2010.03.002.

(19) Yilmaz, H.M., Yakar, M., Gulec, S. A., Dulgerler, O. N. (2007). Importance of digital close- range photogrammetry in documentation of cultural heritage. Journal of Cultural Heritage, 8(4): 428-433, doi: http://dx.doi.org/10.1016/j.culher.2007.07.004.

(20) Martinez, S., Ortiz, J., Gil, M. L., Rego, M. T. (2013). Recording Complex Structures Using Close Range Photogrammetry: The Cathedral Of Santiago De Compostela. Photogrammetric Record, 28(144): 375-395, doi: http://dx.doi.org/10.1111/ phor.12040.

(21) Santa Cruz, J. (2003). La fotogrametría digital en el levantamiento de planos de edificios. Informes de la construcción 55(488): 31-40, doi: http://dx.doi.org/10.3989/ic.2003.v55.i488.539.

(22) Autodesk 123D. http://www.123dapp.com/.

(23) Blender. http://www.blender.org/.

(24) Dassault Systmes. http://www.3ds.com/.

(25) Waldhäusl, P., Ogleby, C. (1994). Rules for simple photogrammetric documentation of architecture. En Fryer, J. G. (Ed.) Close Range Techniques and Machine Vision, Symposium of Commission V of ISPRS (pp. 426-429). Melbourne, Australia.

(26) Chandler, J.H., Fryer, J. (2011). Accuracy of Autodesk 123-D Catch? http://homepages.lboro.ac.uk/ cvjhc/OtherFiles/ Accuracy\%20of\%20123DCatch.htm.

(27) Stamatopoulos, C., Fraser, C. S., Cronk, S. (2012). Accuracy aspects of utilizing RAW imagery in phtogrammetric measurement. International Archives of the Photogrammetry, Remote Sensing and Spatial Information Sciences. XXII ISPRS Congress (pp. 387-392). Melbourne, Australia. 